BULL. AUSTRAL. MATH. SOC.

VOL. 33 (1986), 1-20.

\title{
DIRECTIONAL WAVE FRONTS OF \\ REACTION-DIFFUSION SYSTEMS
}

\author{
J. SABINA
}

In this work, we study types of undulatory solutions, that we term Directional Wave Fronts (DWF), of non scalar reaction diffusion systems. The DWFs are a natural extension of the well known Plane Wave Fronts (PWFs) solutions. However, the DWFs admit a certain type of boundary conditions. In the present work we show, under suitable conditions on the reaction term, that DWFs also exhibit typical behaviour of PWFs: we just prove the existence of heteroclinic, homoclinic and periodic families of DWFs. Essentially, we require the reaction term to be linearly uncoupled. These results are the generalization of a previous work, concerning the scalar case.

\section{0 . Introduction}

In this article we study a particular type of solution, with propagatory character, of reaction-diffusion systems:

Received 11 April 1985. I wish to thank Professor J. Fraile for introducing me to the topics here developed, as well as for several work sessions on them.

Copyright Clearance Centre, Inc. Serial-fee code: 0004-9727/86 $\$ A 2.00+0.00$. 


$$
\frac{\partial u}{\partial t}=0 \cdot \Delta u+f(u) \quad u \in \mathbb{R}^{m}, m>1 .
$$

An important aspect in the theory of $R-D$ systems such as $(0)$, is the study of those particular solutions which generate either stable asymptotic states or transitory states of equation (0) (see [7]). The well-known Plane Wave Fronts (PWFs) solutions belong to this group of particular solutions (see [7], [13], [14]). The PWFs are solutions with propagatory properties and exhibiting - in the most significant cases - a typical behaviour; they connect solutions which are stationary and homogeneous states of $(0)$. Moreover, in unidimensional media, those connections are stable asymptotic states of certain initial value problems (see [2], [7], [8]). In other cases, the relevant property of PWFs in applications is the periodicity in space and time (see [10], [13]).

The type of solutions analyzed in this work are the Directional Wave Fronts (DWFs), which are, to some extend, a generalization of PWFs. However, the DWFs have the interesting property of admitting a certain type of boundary condition (see section 1).

The objective of this paper is to extend, to the non-scalar case $(m>1)$, the analysis of the existence and asymptotic behaviour of small amplitude DWFs, developed in previous works for the scalar case (see [9], [16]).

In Section 1, we define the homogeneous Dirichlet and Neumann problems for DWFs. Section 4 contains the main results. There we establish the existence of homoclinic, heteroclinic and periodic DWFs with small amplitude - for the homogeneous Neumann problem. However, it requires certain structure conditions on the reaction term $f$. Essentially, we ask that the linearization of equation ( 0 ) be uncoupled (see Section 2).

In Section 3 we describe the method - a centre manifold theorem used in the analysis of section 4 . This method provides a reduction in the dimension of the problem. It is important to point out that the presence in (0) of drift terms (see [7]), gives an interesting asymptotic behaviour. In this case, such asymptotic behaviour is governed by an ODE which is reversible in the time variable (see section 3). 


\section{Reaction diffusion systems}

Specifically, in this work we deal with the equation:

$$
\frac{\partial u}{\partial t}=D \cdot L u+f(u)
$$

where

$$
L=\Delta+\sum_{i=1}^{n} b_{i} \frac{\partial}{\partial x_{i}} \quad b_{i} \in \mathbb{R}
$$

and where $\Delta=\sum_{i=1}^{n} \frac{\partial^{2}}{\partial x_{i}^{2}}, b=\left(b_{1}, b_{2}, \ldots, b_{n}\right) \in \mathbb{R}^{n}-\{0\}$. In the equation (1) $u: \Omega \times R \rightarrow \mathbb{R} \mathbb{R}^{m}, m>1, \Omega$ being a $C^{2}$ bounded domain in $\mathbb{R}^{m}$. $(x, t) \rightarrow u(x, t)$

The diffusivities matrix $D$ is symmetric and positive definite. $L$ acts coordinate to coordinate: $L u=\left(L u_{i}\right)_{1 \leq i \leq m}$ and the reaction term $f$ is a smooth nonlinear function of $u$ (see section 2).

Some relevant information has been obtained when $b=0$ in (1), and this equation is scalar (see section $5 c$ ), but we do not discuss this case here.

\section{Directional wave fronts. Boundary conditions}

A Directional Wave Front (DWF) is a solution $u=u(x, t)$ of (1) with the form:

$$
u(x, t)=v(K x-c t) \quad x \in \Omega,
$$

where the matrix $K=\operatorname{diag}\left[k_{1}, \ldots, k_{n}\right]$ and $c=\left(c_{1}, \ldots, c_{n}\right) \in \mathbb{R}^{n}$. Thus, $y=K x-c t \in \mathbb{R}^{n}$ if $x \in \Omega$ and $t \in \mathbb{R}$.

It is easy to see that DWFs represent perturbations, which propagate with constant velocity in the direction $K^{-1}$. If $n=1$, PWFs and DWFs are the same type of solution of (1).

In the sequel we assume, for simplicity, that $D=1$. So every DWF $v=v(y)$ of (1) satisfies the equation:

$$
\sum_{i=1}^{n} k_{i}^{2} \frac{\partial^{2} v}{\partial x_{i}^{2}}+\sum_{i=1}^{n}\left(k_{i} b_{i}+c_{i}\right) \frac{\partial v}{\partial x_{i}}+f(v)=0
$$


where the variable $y$ ranges over the unbounded domain:

$$
W=\underset{t \in \mathbb{R}}{U}(K \Omega-c t),
$$

if $x \in \Omega$ and $t \in \mathbb{R}$.

We are interested just in bounded solutions of (1.2) defined on $W$. However, establishing the existence of those solutions and determining their asymptotic behaviour in $W$, may be very hard.

In this work, the approach to the problem consists in transforming it into an abstract evolution problem. In fact, there exists a non singular Iinear transformation $S: \mathbb{R}^{n} \rightarrow \mathbb{R}^{n}$ (see [9], [16]), which puts (1.2) into $y^{\prime} \rightarrow y=S y^{\prime}$

the form:

$$
\frac{\partial^{2} v}{\partial s^{2}}+\Delta z+f(v)=0 \quad s \in \mathbb{R}, z \in D .
$$

In that equation we write $y^{2}=(s, z)$ with $z \in \mathbb{R}^{n-1}$. We are supposing also that the identity $K b+c=0$ holds, hence, the condition $\dot{b} \neq 0$ is essential. If $y \in W$ then $z \in D$, a domain in $\mathbb{R}^{n-1}$. The regularity of $D$ does not depend only on the intrinsic regularity of $\Omega$, but also on $c$ and $K$ (see [9], [16]). Here, we suppose that $\Omega, K$ and $c$ have been chosen in crder to obtain a regular domain $D \subset \mathbb{R}^{n-1}$.

As a consequence of the above discussion, it is possible to impose boundary conditions on DWFs. In fact, by using the transformations $S$ and $K$, the following fact holds (see [9], [16]):

$$
\text { II } \subset \delta \Omega \text { such that } x \in \Gamma \Leftrightarrow z \in \partial D \text {. }
$$

We define the homogeneous Dirichlet problem for DWFs as the finding of solutions $u=u(x, t)$ of (1), with the form (1.1), satisfying:

$$
u(x, t)=0 \quad \text { on } x \in \Gamma, t \in \mathbb{R} \text {. }
$$

On the other hand, a suitable field $\nu=\nu(x)$ can be defined on $\Gamma$ (see [9], [16]) such that, for a DWF $u=u(x, t)$ :

$$
\frac{\partial u}{\partial v}(x, t)=0 \text { on } x \in \Gamma \text { and } t \in \mathbb{R} \Leftrightarrow
$$




$$
\frac{\partial v}{\partial n}(s, z)=0 \text { on } z \in \partial D \text { and } s \in \mathbb{R} \text {. }
$$

Above $n=n(z)$ denotes the outer unitary normal at $z \epsilon \partial D$. Thus, the homogeneous Neumann problem for DWFs is to find $u=u(x, t)$, of type (1.1), such that:

$$
\frac{\partial u}{\partial v}(x, t)=0 \text { on } x \in \Gamma, t \in \mathbb{R} \text {. }
$$

Accordingly, the Dirichlet and Neumann problems for DWFs are, respectively, equivalent to the evolution problems:

$$
\begin{aligned}
& \left(P_{D}\right)\left\{\begin{array}{l}
\frac{\partial^{2} v}{\partial s^{2}}+\Delta_{z} v+f(v)=0 \quad z \in D \\
v=0 \quad z \in \partial D
\end{array}\right. \\
& \left(P_{N}\right) \quad\left\{\begin{array}{l}
\frac{\partial^{2} v}{\partial s^{2}}+\Delta_{z} v+f(v)=0 \quad z \in D \\
\frac{\partial v}{\partial n}=0 \quad z \in \partial D .
\end{array}\right.
\end{aligned}
$$

In this work we shall analyze the Neumann problem. The corresponding conclusions for $\left(P_{D}\right)$ follow in a similar way (see section 5b).

Finally, observe that the only PWFs solutions of (1) satisfying the condition (1.3) are the trivial ones: $u \equiv 0$. Analogously, if a PWF satisfies (1.4) it must be constant in space and time.

\section{The reaction term structure. Consequences.}

We are going to consider the analysis of bounded DWFs as a bifurcation problem, with respect to the solution $u=0$ and a real parameter $\lambda$. Thus, we shall suppose that $f: \mathbb{R} \times \mathbb{R}^{m} \rightarrow \mathbb{R}^{m}$ is a $c^{r+2}$ function, which $(\lambda, u) \rightarrow f(\lambda, u)$

satisfies:

(i) $f(\lambda, 0)=0, \lambda \in \mathbb{R}$. Hence, $f=f(\lambda, u)$ can be written in the form: $f(\lambda, u)=A(\lambda) u+g(\lambda, u)$, $A(\lambda)$ being an $m \times m$ matrix and $g(\lambda, u)=o\left(|u|^{2}\right.$, when $|u| \rightarrow 0$, uniformly in $\lambda,|\lambda|$ small. We suppose also that: 
(ii) $\mathrm{g} a>0$ such that $A(\lambda)=\operatorname{diag}\left[d_{1}(\lambda), \ldots, d_{m}(\lambda)\right], d_{1}(\lambda)>d_{j}(\lambda)$ for $j \in\{2, \ldots, m\}$ and $|\lambda|<a$, with $d_{1}(0)=0$ and $d_{1}(0)>0$. (iii) $g_{1}(\lambda, u)=\sum_{|\alpha|=k} a_{k}^{\alpha}(\lambda) u+r(\lambda, u), k \leq r+1$, where $r(\lambda, u)=o\left(|u|^{k+1}\right)$, when $|u| \rightarrow 0$, uniformly in $\lambda$. If $\alpha_{0}=(k, 0, \ldots, 0) \in N^{m}$ then $a_{k}^{\alpha_{0}}(\lambda) \neq 0$ for $|\lambda|<a$.

REMARKS. Amongst (i), (ii), (iii) the essential hypothesis is (ii). There, we impose the condition that (1) is linearly uncoupled. This condition holds, for example if $f(\lambda, u)=\frac{\partial \phi}{\partial u}(\lambda, u)$, with $\phi=\phi(\lambda, u)$ a scalar smooth function. In general, (i) always implies (iii) with $k=2$. In (i), (ii), (iii) we endow the first equation of (1) with a structure which is analogous to the structure that generates small amplitude DWFs in the scalar case (see [9], [16]).

On the other hand, the presence of smaill amplitude stationaryhomogeneous solutions of (1), suggests the existence of DWFs whose $\alpha$ and $\omega$-limit sets consist of those solutions. The following result ensures the existence of that type of solution.

PROPOSITION 1. Let us consider the kinetic equation associated with (1):

$$
\frac{d u}{d t}=f(\lambda, u)
$$

where $f$ satisfies (i), (ii), (iii). Then:

(1) $(\lambda, u)=(0,0)$ is a bifurcation point of stationary solutions of (2. 1 ) with respect to $u=0$. The bifurcated branch has the form $(\lambda, u)=\left(\lambda\left(u_{1}\right), \phi\left(u_{1}\right)\right), u_{1}$ small, $\phi$ and $\lambda$ being $c^{p+1}$ functions., $\phi_{1}\left(u_{1}\right)=u_{1}, \phi_{j}\left(u_{1}\right)=o\left(u_{1}^{2}\right)$ for $2 \leq j \leq m$, and $\lambda\left(u_{1}\right)=\frac{a_{k}^{\alpha_{0}}(0)}{d_{1}(0)} u_{1}^{k-1}+0\left(\left|u_{1}\right|^{k}\right)$; (2) at $(\lambda, u)=(0,0)$ a stability interchange happens between the bifurcated branches.

Proof. The proof of (1) is an immediate consequence of Theorem 1 in 
[5]. However, because of considerations in Section 4, we give a proof using the Lyapunov-Schmidt method. Let us put $a=\left(u_{2}, \ldots, u_{m}\right)$ and $\hat{A}(\lambda)=\operatorname{diag}\left[d_{2}(\lambda), \ldots, d_{m}(\lambda)\right]$, then equation $f=0$ can be written in the form

$$
\left\{\begin{array}{l}
d_{1}(\lambda) u_{1}+g_{1}\left(\lambda, u_{1}, \hat{u}\right)=0, \\
\hat{A}(\lambda) \hat{u}+\hat{g}\left(\lambda, u_{1}, \hat{u}\right)=0 .
\end{array}\right.
$$

The implicit function theorem, applied to the second equation, implies that $\hat{u}=\psi\left(\lambda, u_{1}\right)$, where $\psi$ is a $c^{p+2}$ function satisfying: $\psi\left(\lambda, u_{1}\right)=0\left(\left|u_{1}\right|^{2}\right)$, when $\left|u_{1}\right| \rightarrow 0$ and $|\lambda|$ small.

Hence, (2.2) is equivalent to:

$$
d_{1}(\lambda) u_{1}+g_{1}\left(\lambda, u_{1}, \psi\left(\lambda, u_{1}\right)\right)=0 \text {. }
$$

and nontrivial solutions of $(2.2)$ are just $(\lambda, u)=\left(\lambda\left(u_{1}\right),\left(u_{1}, \psi\left(\lambda, u_{1}\right)\right)\right)$, where $\lambda(\cdot)$ is the $c^{p+1}$ function defined by the equation:

$$
d_{1}(\lambda)+a_{k}^{\alpha}(\lambda) u_{1}^{k-1}+\tilde{r}\left(\lambda, u_{1}\right)=0
$$

where $\tilde{r}\left(\lambda, u_{1}\right)=0\left(\left|u_{1}\right|^{k}\right)$, when $\left|u_{1}\right| \rightarrow 0,|\lambda|$ small.

(2) is a well known fact from bifurcation theory (see [11]).\#

The abstract approach to the evolution problem $\left(P_{N}\right)$ in a Hilbert space frame, requires an additional condition, on $f$ namely:

(iv) $f$ together with its derivatives up to the order $r+1$, are polynomially bounded. Also we assume that $n \leq 3$.

From (iv) the function $f$ is smooth, considered as a Nemitskii

operator in $\left(H^{l}(D)\right)^{m}$ with values in $\left(L^{2}(D)\right)^{m}$ :

PROPOSITION 2. Assume that $f \in C^{r+2}\left(\mathbb{R} \times \mathbb{R}^{m}, \mathbb{R}^{m}\right)$ and suppose that (iv) holds, that is, $n \leq 3$ and:

$$
\left|\partial^{\gamma} f(\lambda, u)\right| \leq b_{\gamma}(\lambda)+c_{\gamma}(\lambda) \sum_{j=1}^{m}\left|u_{j}\right|^{s} \gamma, j,
$$


with $\gamma \in N^{m+1}, 0 \leq|\gamma| \leq r+1, s_{\gamma, j} \geq 1, b_{\gamma}(\cdot)$ and $c_{\gamma}(\cdot)$

continuous. Then :

$$
f \in C^{r+1}\left(\mathbb{R} \times\left(H^{l}(D)\right)^{m},\left(L^{2}(D)\right)^{m}\right) .
$$

Proof. If $\Omega \subset \mathbb{R}^{3}$ then $D \subset \mathbb{R}^{2}$. Thus, sobolev Immersions implies that $\left(H^{1}(D)\right)^{m} \subset\left(I^{p}(D)\right)^{m}, \forall p>1$, with compact embedding. Then, it suffices to prove that $f_{i} \in C^{p+1}\left(\mathbb{R} \times\left(H^{1}(D)\right)^{m}, L^{p}(D)\right)$, for some $p>2$. Let us take $u=\left(u_{1}, \ldots, u_{m}\right)$ and $h=\left(h_{1}, \ldots, h_{m}\right) \in\left(H^{1}(D)\right)^{m}$. A.t every $z \in D$ we can write:

$f_{i}(\lambda+\hat{\lambda}, u(z)+h(z))=\sum_{\ell=0}^{r+1} \frac{1}{\ell !} \sum_{k=0}^{\ell}\left(\begin{array}{l}\ell \\ k\end{array}\right)\left(\partial_{\lambda}^{\ell-k} \partial_{u}^{k} f_{i}(\lambda, u(z)) h^{k}(z)\right) \lambda^{\ell-k}+\rho(\hat{\lambda}, h(z))$, where

$$
\rho(\hat{\lambda}, h(z))=\frac{1}{(r+2) !} \sum_{k=0}^{r+2}\left(\begin{array}{c}
r+2 \\
k
\end{array}\right) \partial_{\lambda}^{r+2-k}\left(\partial_{u}^{k} f_{i}(\lambda+\theta(z) \hat{\lambda},(u+\theta h)(z)) h^{k}(z)\right) \hat{\lambda}^{r+2-k}
$$

and where $0<\theta(z)<1$, a.e. in $D$.

Because of the polynomial growth of $\partial^{\gamma} f(\lambda, u),|\gamma|=k \leq r \leq 1$, then $\partial_{u} f_{i}(\cdot, \cdot) \in C\left(\mathbb{R} \times\left(L^{p}(D)\right)^{m}, L^{k}\left(\left(L^{p}(D)\right)^{m}, L^{2}(D)\right)\right)$, for $\left.p \geq \underset{\substack{|\gamma|=k \\ 1 \leq j \leq m}}{\max \{2 s, j}\right\}$ (see [3]). For $X=\left(L^{p}(D)\right)^{m}$ and $Y=L^{2}(D), L^{k}(X, Y)$ denotes the space of the continuous $k$-linear maps from $X$ to $Y$.

On the other hand:

$$
o(\hat{\lambda}, h)=o\left(\left(|\hat{\lambda}|+|h|_{p}\right)^{r+2}\right),
$$

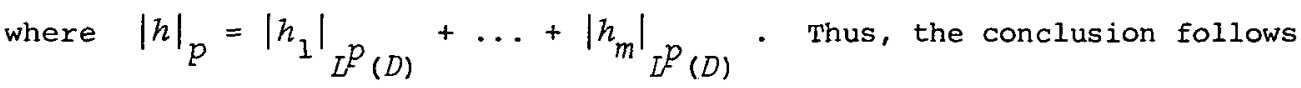
from the Converse Taylor's Theorem (see [1]).\#

3. A reduction in the dimension of the problem In the study of small amplitude solutions of $\left(P_{N}\right)$, a reduction in the dimension can be introduced. Here, the information about those solutions, is furnished by a two-dimensional O.D.E. Let us describe how 
such a reduction can be performed.

Firstly, the problem $\left(P_{N}\right)$ can be rewritten in the form:

$$
v^{\prime \prime}-T(\lambda) v+g(\lambda, v)=0 \quad \cdot=\frac{d}{d s},
$$

where $T(\lambda)=-\Delta_{z}-A(\lambda)$ is defined in $\left(L^{2}(D)\right)^{m}$, with domain:

$$
D(T)=\left\{v \in\left(H^{2}(D)\right)^{m} / \frac{\partial v}{\partial n}=0 \text { on } \partial D\right\}
$$

The operator $T(\lambda)$ has compact resolvent, so its spectrum

$\Sigma(T(\lambda))=\bigcup_{i=1}^{m}\left\{\sigma_{k}-d_{i}(\lambda)\right\}_{k \in N},\left\{\sigma_{k}\right\}_{k \in N}$ being the eigenvalue sequence of $-\Delta$ defined on $L^{2}(D)$, with domain

$$
D=\left\{v \in H^{2}(D) / \frac{\partial v}{\partial n}=0 \text { on } \partial D\right\} \text {. }
$$

Thus, for $|\lambda|$ small enough, $\mu=0$ is the first eigenvalue of $T(\lambda)$, with corresponding eigenfunction $\psi=1_{D} e_{1}, e_{1}=(1,0, \ldots 0) \in \mathbb{R}^{m}, 1_{D}(z)=1$, $\forall z \in D$. If $H_{0}=\operatorname{span}\{\psi\}, H_{1}=\left(H_{0}\right)_{\left(L^{2}(D)\right)^{m}}^{\frac{1}{m}}$ then $L^{2}(D)=H_{0} \oplus H_{1}$.

Denoting by $P_{i}$ the projection onto $H_{i}$, and writing $T_{i}(\lambda)=\left.P_{i} \circ T(\lambda)\right|_{H_{i}}, i=0,1$, we can put (3.1) in the form:

$$
\left\{\begin{array}{l}
v_{0}^{\prime \prime}+d_{1}(\lambda) v_{0}+\tilde{g}_{0}\left(\lambda, v_{0}, v_{1}\right)=0 \\
v_{1}^{\prime \prime}-T_{1}(\lambda) v_{1}+\tilde{g}_{1}\left(\lambda, v_{0}, v_{1}\right)=0
\end{array}\right.
$$

Above, for $v \in\left(L^{2}(D)\right)^{m}, v=v_{0} e_{1}+v_{1}=P_{0} v+P_{1} v$ and, for example, $P_{0} g\left(\lambda, v_{0} e_{1}+v_{1}\right)=\tilde{g}_{0}\left(\lambda, v_{0}, v_{1}\right) e_{1}$, where:

$$
\tilde{g}_{0}\left(\lambda, v_{0}, v_{1}\right)=\frac{1}{|D|} \int_{D} g_{1}\left(\lambda, v_{0}(z) e_{1}+v_{1}(z)\right) d z
$$

with $v \in\left(H^{l}(D)\right)^{m}$ and $|D|$ being the Lebesgue measure of $D \subset \mathbb{R}^{2}$ Equation (3.2) exhibits a coupling between a bidimensional equation $((3.2 .1))$ and a infinite dimensional one ((3.2.2)). We shall show how 
(3.2.1) it is just the result of the behaviour of small amplitude solutions of (3.1).

Intuitively, observe that, for $\lambda$ small:

$$
\exists \alpha>0:\left(T_{1}(\lambda) v, v\right) \geq \alpha|v|_{\left(L^{2}(D)\right)^{m}}^{2}, v \in D\left(T_{1}\right)
$$

where $(\cdot, \cdot)$ denotes the usual $\left(L^{2}(D)\right)^{m}$ inner product. Therefore, the linear part of (3.2.2) has its spectrum bounded away from Rez $=0$. However, the corresponding spectrum of (3.2.1) is $\left\{ \pm \sqrt{d_{1}(\lambda)}\right\}$ which collapses to $\{0\}$ when $\lambda \rightarrow 0$. This fact suggests the existence of $a$ Centre Manifold (see [4]). In fact, a theorem of existence of Center Manifolds, due to Kirchgassner (see [12]), for abstract evolution equations of type (3.1) is applicable. This result implies the existence of $\varepsilon_{0}$, $\varepsilon_{1}, \varepsilon_{2}$, positive numbers and a smooth function:

$$
h \in C^{r}\left(\left(-\varepsilon_{0}, \varepsilon_{0}\right) \times B_{\varepsilon_{1}}^{2}(0), B_{\varepsilon_{2}}^{\prime}(0)\right)
$$

where $B_{\varepsilon_{1}}^{2}(0)$ is the open ball in $\mathbb{R}^{2}$, centred at 0 with radius $\varepsilon_{1}>0$, and $B_{\varepsilon_{2}}^{\prime}(0)$ the corresponding ball in the space $\left(H^{1}(D)\right)^{m} \cap H_{1}$. The function $h$ - known in the literature as a Centre Manifold (see [4]) has the following invariance property: If $v=v(s)$ is a solution of (3.1), $|\lambda|<\varepsilon_{0}$, with the regularity $v \in C^{2}\left(\mathbb{R},\left(L^{2}(D)\right)^{m}\right) \cap C^{1}\left(\mathbb{R},\left(H^{1}(D)\right)^{m}\right)$ $\cap C\left(\mathbb{R},\left(H^{2}(D)\right)^{m}\right)-\operatorname{see}[6$ Chap. 6,55$]$ and $[12]-$ and satisfies

$$
\sup _{s \in \mathbb{R}}\left(v_{0}^{2}(s)+v_{0}^{\prime 2}(s)\right)<\varepsilon_{1}^{2}, \sup _{s \in \mathbb{R}}\left|v_{1}(s)\right|_{\left(H^{1}(D)\right)^{m}}<\varepsilon_{2},
$$

then, such a solution satisfies:

$$
v_{1}(s)=h\left(\lambda, v_{0}(s), v_{0}^{\prime}(s)\right) \quad \forall s \in \mathbb{R} \text {. }
$$

As a consequence, every small amplitude solution of (3.I), regular enough, will has the form: $v(s)=v_{0}(s) e_{1}+h\left(\lambda, v_{0}(s), v_{0}^{\prime}(s)\right)$, where $v_{0}=v_{0}(s)$ is a solution of the equation: 
(3.5)

$$
v_{0}^{\prime \prime}+d_{1}(\lambda) v_{0}+\tilde{g}_{0}\left(\lambda, v_{0} e_{1}+h\left(\lambda, v_{0}, v_{0}^{\prime}\right)\right)=0
$$

Hence, (3.5) governs the small amplitude solutions of $\left(P_{N}\right)$.

Furthermore, function $h$ exhibits the properties (see [4], [12]):

$$
h\left(\lambda, v_{0}, v_{0}^{\prime}\right)=0\left(v_{0}^{2}+v_{0}^{, 2}\right)
$$

$$
h\left(\lambda, v_{0},-v_{0}^{\prime}\right)=h\left(\lambda, v_{0}, v_{0}^{\prime}\right) .
$$

Hence, some information about the nonlinearity in (3.5) can be deduced. In fact, some calculations show that (3.5) can be put in the form:

$$
v_{0}^{\prime \prime}+a(\lambda)_{0}+b(\lambda) v_{0}^{k}+r_{1}\left(\lambda, v_{0}, v_{0}^{\prime}\right)=0
$$

where $a(\lambda)=d_{1}(\lambda), b(\lambda)=a_{k}^{\alpha_{0}}(\lambda)$ and $r_{1}\left(\lambda, v_{0},-v_{0}^{\prime}\right)=r_{1}\left(\lambda, v_{0}, v_{0}^{\prime}\right)$ with $r=0\left(\left|v_{0}\right|^{k+1}+\left|v_{0}^{\prime}\right|^{2}\left|v_{0}\right|^{k-1}\right)$.

REMARKS .

1. The regularity required on $g$ for obtaining $c^{r}$ class in $h$ is just $g \in C^{r+1}\left(\mathbb{R} \times\left(H^{\prime}(D)\right)^{m},\left(L^{2}(D)\right)^{m}\right)$ (see [12]). Thus, if $f=f(\lambda, h)$ satisfies (i), ..., (iv), that condition holds.

2. Every small solution $v_{0}=v_{0}(s)$ of (3.8) generates a DWF $u=u(x, t)$ of equation (3.4), which is solution of the Neumann problem. The regularity of $v(s)=v_{0}(s) e_{1}+h\left(\lambda, v_{0}(s), v_{0}^{\prime}(s)\right)$ implies that $u(\cdot, t) \in H^{2}(\Omega) \forall t \in I R_{c}$ and $u \in H_{l \circ c}^{2}(\Omega \times \mathbb{R})$. Hence, $u(\cdot, t) \in C^{0}(\bar{\Omega}) \forall t \in I R\left(\Omega \subset R^{3}\right)$.

4. Existence of small amplitude DWFs for the Neumann problem Let us begin studying the small amplitude solutions of (3.8). If we consider the truncated equation:

$$
v^{\prime \prime}+a(\lambda) v_{0}+b(\lambda) v_{0}^{k}=0,
$$

via the first integral $v\left(v_{0}, v_{0}^{\prime}\right)=\frac{1}{2} v_{0}^{\prime 2}+a(\lambda) \frac{v_{0}^{2}}{2}+b(\lambda) \frac{v_{0}^{k+1}}{k+1}$, is easily seen that: 
(a) If $k$ is even and $\lambda<0$; then $\left(v_{0}, v_{0}^{\prime}\right)=(0,0)$ is a saddle point of (4.I) connected with itself by a homoclinic orbit, which encloses to the nonlinear center $\left(-(a(\lambda) / b(\lambda))^{1 / k-1}, 0\right)$. Thus, a one parameter family of periodic solutions of (4.1) arises.

If $\lambda>0$, points $(0,0)$ and $\left(-(a(\lambda) / b(\lambda))^{1 / k-1}, 0\right)$ interchange their behaviour (see Figure 1).

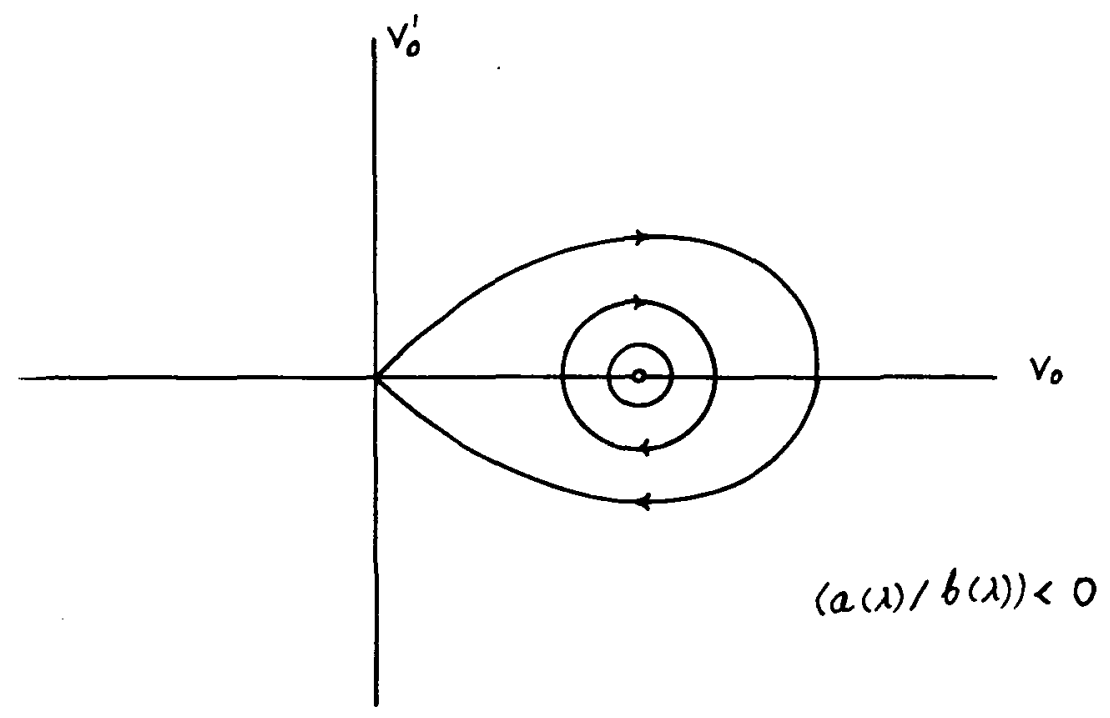

Figure 1.

(b) If $k$ is odd, $\lambda b(\lambda)<0$ and $\lambda<0,(0,0)$ is a saddle point connected with itself by two homoclinic orbits, enclosing the nonlinear centres $\left( \pm(-a(\lambda) / b(\lambda))^{1 / k-1}, 0\right)$. If $\lambda>0,\left( \pm(-a(\lambda) / b(\lambda))^{1 / k-1}, 0\right)$ are saddle points which are connected by two heteroclinic orbits, bounding a domain containing the nonlinear centre $(0,0)$ (see Figure 2).

Let us observe that orbits in a) and $b), \lambda>0$, are all symmetric with respect to the reflection $R_{1}$, with $R_{1}\left(v_{0}, v_{0}^{\prime}\right)=\left(v_{0},-v_{0}^{\prime}\right)$.

Property (3.7) of $h$ implies that (3.8) inherits that symmetry. The results of Renardy in [15] are then applicable and (3.8) inherits also the phase plane configuration of (4.1), with respect to small solutions. 


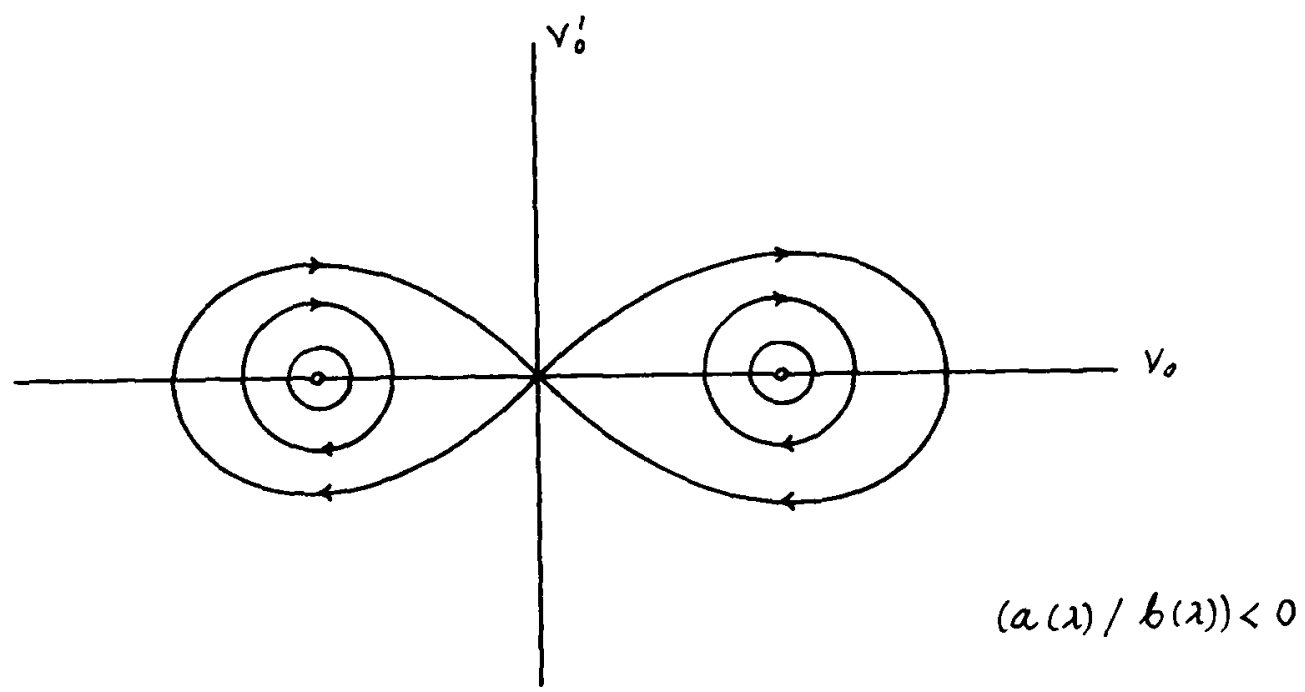

Figure 2.

In the case b), $\lambda>0$, the orbits in (4.1) are $R_{2}$-invariants, with $R_{2}\left(v_{0}, v_{0}^{\prime}\right)=\left(-v_{0}, v_{0}^{\prime}\right)$. Suppose that $f=f(\lambda, u)$ is odd in $u$, in equation ( 1$)$. Then $h$ inherits this property: $h\left(\lambda, v_{0},-v_{0}^{\prime}\right)=-h\left(\lambda, v_{0}, v_{0}^{\prime}\right)$ (see [12]). Hence, (3.8) has this symmetry and Renardy's results ([15]) are again applicable. So, (3.8) has the same plane configuration as (4.1), with respect to small solutions.

The above considerations allow us to ensure, for $|\lambda|$ small enough, the existence of several types of DWFs for the Neumann problem. We sumarize their properties in the next theorem:

THEOREM 3. Assume that conditions (i) ..., (iv) holds and $\lambda \in(-\varepsilon, \varepsilon), \lambda \neq 0$, small enough. Then the Neumann problem for DWFs admits solutions $u=u(x, t)$, with the following properties:

(i) If $k$ is even (respectively odd, $\lambda b(\lambda)<0$ and $\lambda<0$ ), there exists $u=u(\lambda)$, a solution of (2.1) and a DWF $u=u(\lambda, x, t)$ such that:

$$
|u(\lambda, \cdot, t)-u(\lambda)|_{\left(C^{0}(\bar{\Omega})\right)^{m}} \leq C e^{-\beta|+|} \quad t \in \mathbb{R},
$$


where $C>0, \beta>0$ and $u(\lambda)=0$ in $\lambda<0$.

(ii) If $k$ is odd, $f=f(\lambda, u)$ is odd in $u$ and $\lambda b(\lambda)<0$ then, for $\lambda \in(0, \varepsilon)$ there exist $u_{+}(\lambda)$ and $u_{-}(\lambda)$ solutions of (2.1), and a DWF $u=u(\lambda, x, t)$ such that:

$$
\begin{aligned}
& \left|u(\lambda, \cdot, t)-u_{+}(\lambda)\right|_{\left(C^{0}(\bar{\Omega})\right)^{m} \leq C_{+} e^{-\beta} 1 t \quad t \rightarrow \infty,} \\
& \left|u(\lambda, \cdot, t)-u_{-}(\lambda)\right|_{\left(C^{0}(\bar{\Omega})\right)^{m} \leq C_{-} e^{\beta} 2 t \quad t \rightarrow-\infty,}
\end{aligned}
$$

where $C_{+}, C_{-}, B_{1}$ and $B_{2}$ are positive constants.

(iii) Furthermore, in the cases (i) and (ii) there exists a small complitude one parcmeter fomily of t-periodic DWFs.

Proof. First of all, observe that (3.8) admits a branch of stationary solutions $\left(\lambda, v_{0}\right)=\left(\lambda\left(v_{0}\right), v_{0}\right),\left|v_{0}\right|$ small, $\lambda(\cdot)$ being a $C^{p}$ function of the form:

$$
\lambda=\frac{b(0)}{a^{\prime}(0)} v_{0}^{k-1}+0\left(v_{0}^{k}\right)
$$

and satisfies:

$$
a(\lambda)+b(\lambda) v_{0}^{k-1}+\frac{r_{1}\left(\lambda, v_{0}, 0\right)}{v_{0}}=0 .
$$

Thus, $\exists \varepsilon>0$ such that: a) If $k$ is even, $\forall \lambda \in(-\varepsilon, \varepsilon), \exists v_{0}(\lambda) \neq 0$ stationary solution of $(3.8)$. b) If $k$ is odd, $\forall \lambda \in(-\varepsilon, \varepsilon)$ such that $\lambda b(\lambda)>0, \exists v_{0}^{+}(\lambda)$ and $v_{0}^{-}(\lambda)$ which are non zero stationary solutions of (3.8). Furthermore, the stability and connection properties of $(0,0)$, $\left(v_{0}(\lambda), 0\right),\left(v_{0}^{ \pm}(\lambda), 0\right)$ are those of the equation (4.1). On the other hand each of the solutions $w=\omega(\lambda)$ generates a stationary DWF $v_{\lambda}=v_{\lambda}(z)$ :

$$
v_{\lambda}=w(\lambda) e_{1}+h(\lambda, w(\lambda), 0)
$$

Let us prove the point (iii). If $p_{\mu}=p(\mu, \lambda, s)$ denotes the family of periodic solutions, with period $\omega(\mu, \lambda)$, associated to a nonlinear centre, then we get the family of DWFs: 


$$
v_{\mu}(s)=p_{\mu}(s) e_{1}+h\left(\lambda, p_{\mu}(s), p_{\mu}^{\prime}(s)\right)
$$

On the other hand: I $n>0, \kappa_{1}, \ldots, \kappa_{n} \in \mathbb{I}^{n}$ such that, every DWF of (1) is written as: $u=u(x, t)=v(s, z)$, with $s=\kappa_{I} x-n t, z_{j}=\kappa_{j} x$, $j \in\{2, \ldots, m\} ; n$ defined by the equality: $S c=n e_{1}$. Thus, $u_{\mu}(\lambda, x, t)=v(s, z)$ is a $t$-periodic DWF, with period $-\frac{\omega(\mu, \lambda)}{\eta}$.

For proving (i), let $|\lambda|$ small and let $p=p(s)$ be a homoclinic of (3.8) satisfying:

$$
\lim _{|s| \rightarrow \infty} p(s)=w(\lambda)
$$

where $w(\lambda)=0$ if $\lambda<0, w(\lambda)=v_{0}(\lambda)$ if $\lambda>0$. Then, $\exists C_{0}>0$ and $\beta_{0}>0$ such that:

$$
|p(s)-w(\lambda)|+\left|p^{\prime}(s)\right|+\left|p^{\prime \prime}(s)\right| \leq c_{0} e^{-\beta_{0}|s|} \quad s \in R .
$$

If $p_{1}(s)=h\left(\lambda, p(s), p^{\prime}(s)\right)$, boundedness of $\frac{\partial h}{\partial v_{0}}, \frac{\partial h}{\partial v_{0}^{\prime}}$ on $B_{\varepsilon_{2}}^{2}(0)$ implies

$$
\left\|p_{1}(s)-w(\lambda)\right\|_{m}+\left\|p_{1}(s)\right\|_{m} \leq c e^{-\beta_{0}|s|} \quad s \in \mathbb{R},
$$

where, for $\phi \in\left(H^{1}(D)\right)^{m},\|\|_{m}=|\phi|_{\left(H^{1}(D)\right)^{m}}$. If $v(s)=p(s) e_{1}+p_{1}(s)$ then:

$$
\max \left\{\left\|v(s)-v_{\lambda}\right\|_{m^{\prime}}\left\|v^{\prime}(s)\right\|_{m}\right\} \leq C_{2} e^{-\beta_{0} s} \quad s \in \mathbb{R},
$$

where $C_{2}>0$.

Let us put $u_{\lambda}(x)=v_{\lambda}\left(\kappa_{2} x, \ldots, \kappa_{n} x\right)$, we are going to estimate:

$$
\left|u(\cdot, t)-u_{\lambda}(\cdot)\right|_{\left(H^{\prime}(\Omega)\right)^{m}} \text {. }
$$

By using the transformation $S$ (see $\delta 1): W=\underset{t \in \mathbb{R}}{U} W_{t}$ with $W_{t}=S(K \Omega-c t)$. To estimate the above norm, we must estimate 


$$
\begin{aligned}
& \left|v(s)-v_{\lambda}\right|_{\left(H^{l}\left(W_{t}\right)\right)^{m}} \text { or, equivalently, the norm: } \\
& \qquad\left|v(s)-v_{\lambda}\right|_{\left(H^{1}(I(t) \times D)\right)^{m} .},
\end{aligned}
$$

where $I(t)=\left[\alpha_{0}-n t, \alpha_{1}-n t\right]$ and:

$$
\alpha_{0}\left(\text { respectively } \alpha_{1}\right)=\inf (\sup )\left\{s \in \mathbb{R} /(s, z) \in S K \Omega \subset \mathbb{R}^{n}\right\} \text {. }
$$

Thus, we have that:

$$
\begin{gathered}
\left|v(s, \cdot)-v_{\lambda}\right|_{\left(H^{1}(I(t) \times D)\right)}^{2}=\int_{I(t)}\left\|v(s)-v_{\lambda}\right\|_{m}^{2} d s+\int_{I(t)} \int_{D}\left|\frac{d v}{d s}(s, z)\right|^{2} d z d s \\
\quad \leq \int_{I(t)}\left(\left\|v(s)-v_{\lambda}\right\|_{m}^{2}+\left\|v^{\prime}(s)\right\|_{m}^{2}\right) d s \leq 2 C_{2}^{2} \int_{I(t)} e^{-2 \beta_{0}|s|} d s .
\end{gathered}
$$

And the last term is bounded by:

$$
\frac{C^{2}}{\beta} \max \left\{\left(e^{2 \beta_{0} \alpha_{1}}-e^{2 \beta_{0} \alpha_{0}}\right),\left(e^{-2 \beta_{0} \alpha_{0}}-e^{-2 \beta_{0} \alpha_{1}}\right)\right\} \text {. }
$$

Thus, there exists $C_{3}>0$ such that:

$$
\left|v(s)-v_{\lambda}\right|_{\left(H^{l}\left(w_{t}\right)\right)^{m}} \leq C_{3} e^{-\beta_{0} \eta|t|} \quad t \in \mathbb{R} .
$$

If $u=u(x, t)$ is the DWF associated to $v=v(s)$ then we can put $u(x, t)=\tilde{v}(y)$, where $\tilde{v}(y)=v\left(S^{-1} y\right)$ with $y=K x-c t$. If $y^{\prime}=S^{-1} y$ :

$$
\begin{aligned}
& \int_{K \Omega-c t}\left|\tilde{v}(y)-\tilde{v}_{\lambda}\right|^{2} d y=|\operatorname{det} S| \int_{W_{t}}\left|v\left(y^{\prime}\right)-v_{\lambda}\right|^{2} d y^{\prime}, \\
& \int_{K \Omega-c t}\left|\nabla_{y} \tilde{v}_{i}(y)\right|^{2} d y=|\operatorname{det} s| \int_{W_{t}}\left|S^{-1} \nabla_{y^{\prime}} v_{i}\left(y^{\prime}\right)\right|^{2} d y^{\prime}, \quad 1 \leq i \leq m .
\end{aligned}
$$

Taking $C_{4}=C_{3}|\operatorname{det} S|^{\frac{1}{2}} \max \left\{1,\left\|S^{-1}\right\|\right\}$ :

$$
\left|\tilde{v}(\cdot)-v_{\lambda}\right|_{\left(H^{1}(K \Omega-c t)\right)^{m}} \leq C_{4} e^{-\beta_{0} \eta|t|} \quad \text { *t } \in \mathbb{R},
$$

hence 


$$
\left|u(\cdot, t)-u_{\lambda}(\cdot)\right|_{\left(H^{1}(\Omega)\right)^{m}} \leq C_{5} e^{-\beta_{0} n|t|} \quad \forall t \in \mathbb{R}
$$

where $C_{5}=C_{4}|\operatorname{det} K|^{-\frac{1}{2}}(\min \{1, A\})^{\frac{1}{2}}, A=\underset{1 \leq i \leq m}{\min }\left\{\frac{1}{k_{i}^{2}}\right\}$.

On the other hand, boundedness of $\frac{\partial^{2} h}{\partial v_{0}^{2}}, \frac{\partial^{2} h}{\partial v_{0} \partial v_{0}^{\prime}}$ and $\frac{\partial^{2} h}{\partial v_{0}^{2}}$ in $B_{2}^{2}(0)$ implies that:

$$
\left\|p_{1}^{\prime \prime}(s)\right\|_{m} \leq C_{1} e^{-2 \beta_{0}|s|} \quad s \in \mathbb{R},
$$

with $C_{1}^{\prime}>0$. So, $\exists C_{2}^{\prime}>0$ such that:

$$
\left|v^{\prime \prime}(s)\right|_{\left(L^{2}(D)\right)^{m}} \leq \| v(s) \mathbb{R}_{m} \leq C_{2} e^{-\beta_{0}|s|} \quad s \in \mathbb{R} \text {. }
$$

On the other hand:

$$
\Delta_{z} v_{\lambda, i}=-f_{i}\left(\lambda, v_{\lambda}\right) \quad 1 \leq i \leq m \text {, }
$$

then, for $1 \leq i \leq m$ :

$$
\Delta_{z}\left(v_{i}(s)-v_{\lambda, i}\right)=-d_{i}(\lambda)\left(v_{i}(s)-v_{\lambda, i}\right)-\left(g_{i}(\lambda, v)-g_{i}\left(\lambda, v_{\lambda}\right)\right)-v_{i}^{\prime \prime}(s),
$$

where $s \in \boldsymbol{R}$. Thus:

$$
\begin{aligned}
\left|\Delta_{z}\left(v_{i}-v_{\lambda, i}\right\rangle\right|_{\left(L^{2}(D)\right)^{m}} & \leq\left|v_{i}^{\prime \prime(s)}\right|_{L^{2}(D)}+\underset{|\lambda|<\varepsilon}{\sup _{i}\left|d_{i}(\lambda)\right|\left|v_{i}(s)-v_{\lambda, i}\right|_{H^{l}(D)}} \\
& +\left|g_{i}(\lambda, v)-g_{i}\left(\lambda, v_{\lambda}\right)\right|,
\end{aligned}
$$

Considering that $g_{i} \in C^{r}\left(\left(H^{\prime}(D)\right)^{m}, L^{2}(D)\right)$ holds, then:

$$
\mid \Delta_{z}\left(v_{i}(s)-v_{\lambda, i}\right){ }_{L}^{2}(D) \leq C_{3}^{\prime} e^{-\min \{1, n\} B_{0}|s|} \quad s \in R \text {. }
$$

with $C_{3}^{\prime}>0$. Because $\left(v_{i}(s)-v_{\lambda, i}\right) \in H^{2}(D)$ and the ellipticity of (4.5), we get the estimate: 


$$
\left|v_{i}(s)-v_{\lambda, i}\right|_{H^{2}(D)} \leq\left(\left|v_{i}(s)-v_{\lambda, i}\right|_{L^{2}(D)}+\left|\Delta_{z}\left(v_{i}(s)-v_{\lambda, i}\right)\right|_{L^{2}(D)}\right)
$$

thus (4.2) follows from the above inequality and the continuous inclusion $H^{2}(D) \subseteq C^{0}(D)$.

Finally, let us show that $u_{\lambda}=u_{\lambda}(x)$ is homogeneous, that is is a stationary solution of (2.1). In fact, if $u(\lambda)$ is a small solution of (2.1) given by Proposition 1 , then $u(\lambda)$ must lie on the centre manifold (see [4]). Therefore, $\exists \tilde{w}(\lambda) \in \mathbb{R}$ a stationary solution of (3.8) such that: $u(\lambda)=\tilde{w}(\lambda) e_{1}+h(\lambda, \tilde{w}(\lambda), 0)$, where $h(\lambda, \tilde{w}(\lambda), 0)$ is homogeneous. Because of the local uniqueness of bifurcated branches of stationary solutions of $(3.8)$ at $\left(\lambda, v_{0}\right)=(0,0)$ (compare the equations (4.4) and $(2.4))$, it follows that $\tilde{w}(\lambda)=w(\lambda)$.

Let us observe that periodic DWFs obtained above are also small in the $\left(C^{0}(\Omega)\right)^{m}$ norm. The proof in the case (ii) is analogous. \#

\section{Final remarks}

(A) Theorem 3 settles the existence of homo- and heteroclinic DWFs of (1), in (i) and (ii) respectively. Moreover, such DWFs connect stable solutions of (2.1). On the other hand, such DWFs approximate - in the $\left(H^{l}(\Omega)\right)^{m}$ norm - to PWFs. Specifically, in the case (i) for example, it is easy to show that:

$$
|u(\cdot, t)-z(\cdot, t)|_{\left(H^{1}(\Omega)\right)^{m}} \leq C^{\prime} e^{-\beta|t|} \quad t \in \mathbb{R},
$$

where

$$
z(x, t)=v_{0}\left(\kappa_{1} x-n t\right) e_{1}+h(\lambda, \omega(\lambda), 0)=\varnothing\left(\kappa_{1} x-t\right)
$$

Observe that $z=z(x, t)$ is a PWF which also exhibits a homoclinic character: it connects the same solution of (2.1) as $u=u(x, t)$. However $z=z(x, t)$ is not a solution of the equation (1).

(B) The Dirichlet problem can be analyzed with the technigues employed in the Neumann problem. Essentially, $\left(H_{0}^{\prime}(D)\right)^{m}$ must replace to $\left(H^{\prime}(D)\right)^{m}$ in 53, and in hypothesis (i), (ii), (iii) must the positiveness be considered 
of the first eigenvalue $\sigma_{0}$ of $-\Delta_{z}$ in $H_{0}^{l}(D)$. In this way, the conclusions of Theorem 3 still hold for the Dirichlet problem. Observe that, the DWFs of points (i) and (ii) now converge to stationary, but not necessarily homogeneous, solutions of (1) (see [16]).

(C) Condition $b \neq 0$ in (1) is essential for the reversibility in $s$ of $\left(P_{D}\right)$ and $\left(P_{N}\right):$ no terms in $v^{\prime}$ appear in those problems. If $b=0$ the identity $K b+c=0$ is not possible with $K$ and $c$ non-zero.

However, when $b=0$ and $m=1$ (that is, the scalar case) we have obtained the existence of heteroclinic DWFs for $\left(P_{N}\right)$ and $\left(P_{D}\right)$. The reaction term structure is:

$$
f(\lambda, u)=a(\lambda) u+a_{k}(\lambda) u^{k}+o\left(|u|^{k+1}\right) .
$$

where $a(0)=0, \frac{d u}{d}(0) \neq 0$ and $a_{k}(0)=0$. This structure in $f$ is precisely the right one for obtaining, in the scalar case, the results of Theorem 3 (see [8] and [16]). However, to eliminate the restriction $b \neq 0$ in the case of non scalar equations, is an open problem.

\section{References}

[1] R. Abraham and J. Robin, Transversal Mappings and Flows, (W.A. Benjamin, New York, 1967).

[2] D. Aronson and H. Weinberger, Nonlinear diffusion in population genetrics, combustion, and nerve impulse propagation (Lecture Notes in Maths. 446, 5-49, Springer, New York, 1975).

[3] M.S. Berger, Nonlinearity and Functional Analysis, (Academic Press, New York, 1977).

[4] S.N. Chow and J.K. Hale, Methods of Bifurcation Theory, (Grund. der Math. 251 Springer, Berlin, 1982).

[5] M.G. Crandall, "An introduction to constructive aspects of bifurcation theory". In Applications of Bifurcation Theory, Rabinowitz Ed. (Academic Press, New York, 1977).

[6] H.O. Fatorini, "The Cauchy Problem", Encyclopedia of Maths. and Appls. 18, (Addison-Wesley Pub. Co., Reading, Massachusetts, 1983). 
[7] P.C. Fife, Mathematical aspects of reacting and diffusing systems (Lecture Notes in Biomaths. 28 Springer, New York, 1979).

[8] P.C. Fife and J.B. McLeod, "A phase plane discussion of convergence to travelling fronts for nonlinear diffusion", Arch. for Rat. Mech. and Analysis 75 (1981), 281-314.

[9] J. Fraile and J. Sabina, "Boundary value conditions for wave solutions of reaction-diffusion systems". Proceedings of the Royal Society of Edinburgh 99A (1984), 127-136.

[10] L.N. Howard, "Chemical wave-trains and related structures". In Applications of Bifurcation Theory, P. Rabinowitz Ed. (Academic Press, New York, 1977).

[11] G. Iooss and D. Joseph, "Elementary Stability and Bifurcation Theory", Undergrad. Texts in Maths., Springer, Berlin, 1980.

[12] K. Kirchgassner, Homocinic bifurcation of perturbed reversible systems (Lecture Notes in Maths., 1017 (1982).)

[13] N. Kopell and L.N. Howard, "Plane wave solutions to reactiondiffusion equations, Stud. Appl. Math. 52 (1973), 291-328.

[14] J.D. Murray, Lectures on Nonlinear Differential Equation Models in Biology, (clarendon Press, Oxford, 1977).

[15] M. Renardy, "Bifurcation of singular and transient solutions". In Recent Contributions to Nonlinear Partial Differential Equations, (Berestycki-Brezis Eds., Pitman, London, 1981).

[16] J. Sabina, Doctoral Thesis. Universidad Complutense de Madrid, Madrid, (1984).

Dpto. de Ecuaciones Funcionales,

Facultad de Matematicas, U. Complutense.

28040-Madrid

and

Dpto. de Matematicas I,

ETSI de Telecomunicación, U. Politecnica de Madrid

28040-Madrid. 